\title{
Schöne neue Datenschutzordnung 2018?!
}
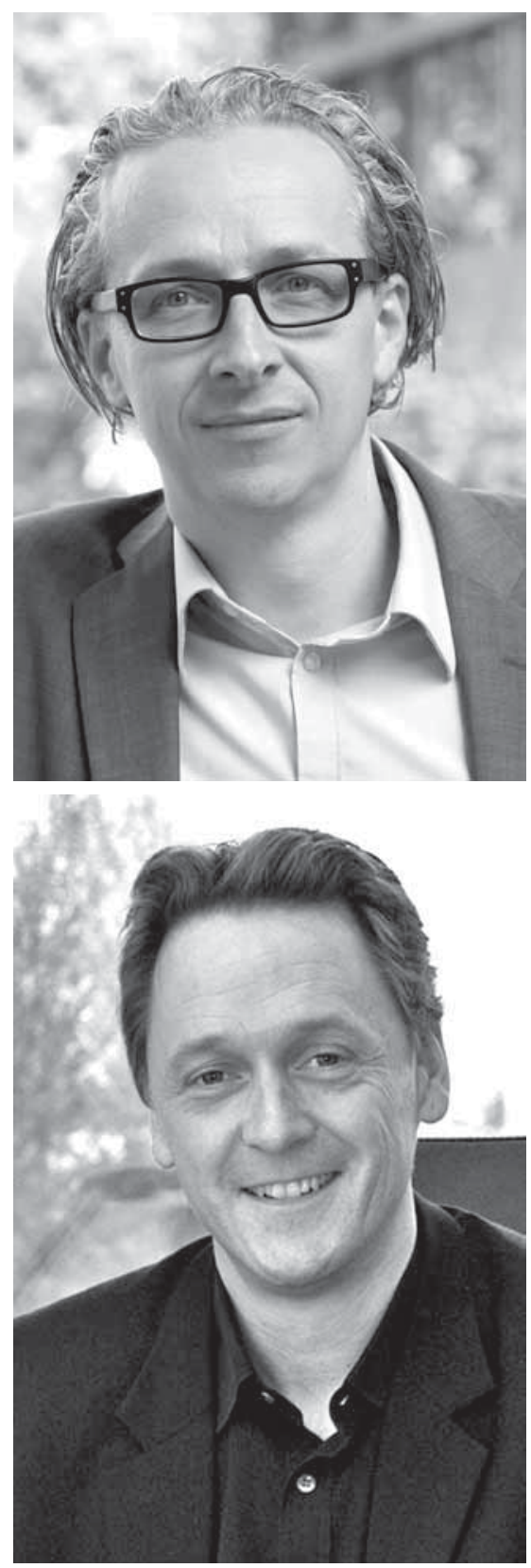

Langsam fügen sich die Puzzleteile zusammen für das neue Bild der Datenschutzordnung, die am 25. Mai 2018 wirksam werden wird - erst die Datenschutz-Grundverordnung vom Mai 2016, ein Jahr später das neue Bundesdatenschutzgesetz. Nun bleibt nicht mal mehr ein Jahr Zeit für die Datenverarbeiter, sich auf diese neue Rechtslage einzustellen. Vieles wird sicherlich besser, etwa der Vollzug des Datenschutzrechts angesichts verschärfter Sanktionen, eines verbesserten institutionellen Rahmens - Stichwort Kohärenzverfahren und Europäischer Datenschutzausschuss - und eines klaren Bekenntnisses zum Marktortprinzip für die Achtung des Datenschutzes auch durch Verarbeiter aus Drittstaaten.

Eines wird die Datenschutzordnung aber sicherlich nicht: einfacher. Das Gegenteil ist vielmehr der Fall. Aufgrund zahlreicher Öffnungsklauseln in der Verordnung wird das Zusammenspiel aus nationaler und supranationaler Ebene deutlich komplizierter. Streitigkeiten über die zutreffende Nutzung der Öffnungsklauseln sind ohnehin vorprogrammiert. Auch der Effekt der Binnenmarktharmonisierung wird nicht so groß sein wie ursprünglich im Kommissionsentwurf der Verordnung geplant. Die Komplexitätssteigerung könnte einen heilsamen Effekt für künftige Reformen der Verordnung haben und die Bereitschaft zum Abbau der Öffnungsklauseln stärken. Unabhängig davon werden auch grundlegende Fragen wie die der Reichweite des Konzepts der Einwilligung und des besonderen Schutzes sensibler Daten oder der Möglichkeiten eines Datenschutzes durch Technik weiter zu diskutieren sein.

Das vorliegende Themenheft will einen Beitrag zu dieser facettenreichen Debatte leisten und vor allem die verschiedenen Probleme auch in der komplexen Mehrebenenperspektive beleuchten. So zeigen sich Unstimmigkeiten zwischen den Ebenen etwa bei der Frage der räumlichen Anwendbarkeit des Datenschutzrechts, die im Beitrag von Manuel Klar adressiert werden. Gleichwohl ist die Einführung des Marktortprinzips sicherlich eine der Errungenschaften der Verordnung. Der Beitrag von Thilo Weichert wirft grundlegend die Frage eines sinnvollen Schutzkonzeptes für sensible Daten auf und sieht massive Defizite in der Herangehensweise des deutschen Gesetzgebers. Die Einwilligung, die in unserem Beitrag besprochen wird, wurde mit der eigenständigen Regelung zu den Bedingungen im Fall der Einwilligung eines Kindes zwar mit der Fokussierung der Dienste der Informationsgesellschaft in der Verordnung modernisiert. Vieles wird gleichwohl auch hier noch zu klären sein. Die in der Praxis besonders bedeutsame Konstellation der Auftragsverarbeitung ist in der DS-GVO abschließend geregelt. Jürgen Hartung und Lisa Büttgen stellen dar, ob und wie dieser rein europäische Regelungsrahmen von den bisherigen Vorgaben des nationalen Rechts für die Auftragsverarbeitung abweicht. Matthias Bergt gibt einen Überblick über die Sanktionsnormen der DS-GVO und geht hierbei vor allem auf die besonders „,teure “ Vorschrift des Art. 83 DS-GVO ein, die für Verstöße Geldbußen von bis zu 4 Prozent des weltweiten Jahresumsatzes androht. Eine zentrale Rolle spielt in der DS-GVO des Weiteren auch der Datenschutz durch Technik. Die DS-GVO hat diesen zwar nicht neu erfunden, präsentiert aber mit Vorgaben wie denen zu Privacy by Design und Privacy by Default oder der Datenschutz-Folgeabschätzung jedenfalls einen konzeptionell geschlossenen Regelungsansatz, wie Silke Jandt in ihrem Beitrag aufzeigt. Annika Kieck und Dirk Pohl gehen schließlich noch einmal auf eine Grundsatzfrage zum Anwendungsbereich der DS-GVO ein und sprechen sich ausdrücklich für eine umfassende Geltung des europäischen Datenschutzrechts auch für rein nationale Sachverhalte aus.

In der Rubrik „Sonstige Beiträge“" wenden sich Kurt Einzinger und Florian Skopik der Schnittstelle zwischen IT-Sicherheit und Datenschutz zu und gehen auf datenschutzrechtliche Fragestellungen rund um sog. Computer Emergency Response Teams (CERTs bzw. CSIRTs) ein. Thema von Sven Venzke-Caprarese in der Rubrik "Good Practice" ist das Retargeting in der Online-Werbung - insoweit gilt dann wieder, dass nach der Reform vor der Reform ist, weil hier mit der geplanten ePrivacyVerordnung schon wieder ein neues europäisches Regelwerk vor der Tür steht.

Wir hoffen mit dieser Themenzusammenstellung Licht und Schatten der Datenschutzordnung 2018 zu konturieren. 